\title{
The distribution, clearance, and brainstem toxicity of panobinostat administered by convection-enhanced delivery
}

\author{
William G. B. Singleton, PhD, MRCS, ${ }^{1}$ Alison S. Bienemann, PhD, ${ }^{1}$ Max Woolley, PhD, ${ }^{1,2}$ \\ David Johnson, PhD, ${ }^{1,2}$ Owen Lewis, BEng, ${ }^{2}$ Marcella J. Wyatt, MSc, ${ }^{1}$ \\ Stephen J. P. Damment, PhD, ${ }^{3}$ Lisa J. Boulter, BSc, ${ }^{1}$ Clare L. Killick-Cole, PhD, ${ }^{1}$ \\ Daniel J. Asby, PhD, ${ }^{1}$ and Steven S. Gill, MS, FRCS ${ }^{1,2}$
}

\begin{abstract}
${ }^{1}$ Functional Neurosurgery Research Group, School of Clinical Sciences, University of Bristol; ${ }^{2}$ Neurological Applications Department, Renishaw PLC, Wotton under Edge, Gloucestershire; and ${ }^{3}$ Midatech Pharma plc, Abingdon, Oxfordshire, United Kingdom
\end{abstract}

\begin{abstract}
OBJECTIVE The pan-histone deacetylase inhibitor panobinostat has preclinical efficacy against diffuse intrinsic pontine glioma (DIPG), and the oral formulation has entered a Phase I clinical trial. However, panobinostat does not cross the blood-brain barrier in humans. Convection-enhanced delivery (CED) is a novel neurosurgical drug delivery technique that bypasses the blood-brain barrier and is of considerable clinical interest in the treatment of DIPG.

METHODS The authors investigated the toxicity, distribution, and clearance of a water-soluble formulation of panobinostat (MTX110) in a small- and large-animal model of CED. Juvenile male Wistar rats $(n=24)$ received panobinostat administered to the pons by CED at increasing concentrations and findings were compared to those in animals that received vehicle alone $(n=12)$. Clinical observation continued for 2 weeks. Animals were sacrificed at 72 hours or 2 weeks following treatment, and the brains were subjected to neuropathological analysis. A further 8 animals received panobinostat by CED to the striatum and were sacrificed 0, 2, 6, or 24 hours after infusion, and their brains explanted and snapfrozen. Tissue-drug concentration was determined by liquid chromatography tandem mass spectrometry (LC-MS/MS). Large-animal toxicity was investigated using a clinically relevant MRI-guided translational porcine model of CED in which a drug delivery system designed for humans was used. Panobinostat was administered at $30 \mu \mathrm{M}$ to the ventral pons of 2 juvenile Large White-Landrace cross pigs. The animals were subjected to clinical and neuropathological analysis, and findings were compared to those obtained in controls after either 1 or 2 weeks. Drug distribution was determined by LCMS/MS in porcine white and gray matter immediately after CED.
\end{abstract}

RESULTS There were no clinical or neuropathological signs of toxicity up to an infused concentration of $30 \mu \mathrm{M}$ in both small- and large-animal models. The half-life of panobinostat in rat brain after CED was 2.9 hours, and the drug was observed to be distributed in porcine white and gray matter with a volume infusion/distribution ratio of 2 and 3 , respectively.

CONCLUSIONS CED of water-soluble panobinostat, up to a concentration of $30 \mu \mathrm{M}$, was not toxic and was distributed effectively in normal brain. CED of panobinostat warrants clinical investigation in patients with DIPG.

https://thejns.org/doi/abs/10.3171/2018.2.PEDS17663

KEYWORDS convection-enhanced delivery; panobinostat; HDAC inhibitor; histone deacetylase inhibitor; toxicity; volume of distribution; half-life; translation; DIPG; brainstem; oncology

$\mathrm{D}$ IFFUSE intrinsic pontine glioma (DIPG) is a highgrade glial malignancy of childhood that arises from the ventral pons. The prognosis is poor; patients have a median survival of approximately 9 months, and, to date, the survival has not been improved with systemic therapy of any kind..$^{14,28}$ Treatment failure is, in part, due to poor CNS penetration of systemically administered drugs due to an intact blood-brain barrier (BBB)..$^{10,19}$

Convection-enhanced delivery (CED) represents a method of direct intraparenchymal administration of drug to the brain via surgically implanted microcatheters. By infusing at precisely controlled, low infusion rates, a drug

ABBREVIATIONS aCSF = artificial CSF; BBB = blood-brain barrier; $C E D=$ convection-enhanced delivery; DIPG = diffuse intrinsic pontine glioma; $\mathrm{LC}$-MS/MS = liquid chromatography tandem mass spectrometry. 
can be distributed over a large volume of brain by bulk flow, displacing the extracellular fluid with infusate. Effective distribution is dependent on many factors, including catheter design and infusion rate, the drug molecule size, drug charge and tissue affinity or binding, drug clearance, and drug water solubility. ${ }^{6,9,20,25}$ CED has therefore been advocated as a novel method of drug delivery for the treatment of DIPG, and it is in early stages of clinical investigation..$^{10,18,27,32}$

Panobinostat is an FDA- and NICE-approved pan-histone deacetylase inhibitor, which has been identified as being effective at reducing DIPG cell viability in vitro and in vivo. ${ }^{11}$ Approximately $80 \%$ of DIPGs have a specific H3 histone mutation, H3K27M, which results in histone hypomethylation and downstream epigenetic changes, supporting the theory that DIPG is epigenetically driven., ${ }^{7,8,16}$, ${ }^{21,31}$ In a study by Grasso et al., panobinostat was the most effective drug at slowing the growth of DIPG cell lines that had this mutation and, importantly, also those that did not. ${ }^{11-13}$ However, the in vivo efficacy may be limited by concerns that panobinostat is toxic at systemic doses that are required to be effective in the CNS. A recent in vivo study using systemically administered panobinostat to mouse models of DIPG failed to show a therapeutic effect, which may be due to poor penetration of the BBB. ${ }^{13}$ Supporting this, published clinical pharmacokinetic data show that it does not cross the BBB in humans and is a substrate of P-glycoprotein, the main drug efflux transporter at the BBB. $\cdot^{13,23,24,29}$

The fact that panobinostat is an efficacious, clinically available drug against DIPG in vitro, that oral dosing may cause unacceptable systemic toxicity, and that it does not penetrate the CNS in humans means that it is of considerable interest as a treatment for DIPG when administered by CED. However, the ability of CED of panobinostat is limited by its poor water solubility. Drugs need to be water soluble at physiological $\mathrm{pH}$ to be delivered to the brain by CED. One method to overcome this limitation is to package the drug in a water-soluble nanoparticle or micelle. ${ }^{26}$ The translation of this nanoparticle formulation of panobinostat to the clinic is challenging, as the breakdown and metabolism of the encapsulating nanoparticle within the brain is unknown, as is its potential for long-term accumulation or toxicity. An alternative method of producing a water-soluble formulation of panobinostat designed for CED may allow a quicker path to the clinic.

We describe a series of in vivo experiments in smalland large-animal models of CED using a water-soluble formulation of panobinostat (MTX110), which may be suitable for CED at potentially chemotherapeutic concentrations. The large-animal model used a juvenile Large White-Landrace cross pig, which has a large gyrencephalic brain that is anatomically similar to that of a human. This provides a robust translatable platform to analyze the following: 1) the toxicity of drug infused in large clinically meaningful volumes and 2) the distribution of drug in white and gray matter with results that can be extrapolated easily to the human brain. This approach generates meaningful translational data to inform the design of clinical infusion protocols. . $^{5,30}$

\section{Methods}

\section{Animal Models of CED}

All animal studies were conducted under appropriate United Kingdom Home Office licenses. Strict adherence to animal welfare was observed at all times, according to best laboratory practice. All protocols were performed in accordance with the UK Animal Scientific Procedures Act 1986.

\section{CED Procedure in Rats}

Juvenile male Wistar rats $(\mathrm{n}=44$; Harlan) weighing between 175 and $250 \mathrm{~g}$ were group-housed in Tecniplast $1500 \mathrm{U}$ cages with irradiated lignocel bedding and sawdust (International Product Supplies Ltd.). The study room was illuminated by fluorescent lighting set to give a cycle of 12 hours of light and 12 hours of darkness and was airconditioned. The ambient temperature was held between $17^{\circ} \mathrm{C}$ and $22^{\circ} \mathrm{C}$.

\section{Brainstem Infusion: Toxicity Analysis}

Animals were individually anesthetized with $2 \%$ inhaled isoflurane in oxygen in an anesthetic chamber and then placed in a stereotactic frame (David Kopf Instruments). Anesthesia was maintained with inhalational $2 \%$ isoflurane/oxygen. The scalp fur was clipped and skin cleaned using alcoholic chlorhexidine. A midline incision from glabella to occiput was made to expose the skull, and a 2-mm burr hole was drilled to accommodate a custommade laser-cut fused silica CED catheter connected to a 10- $\mu$ l Hamilton syringe and rate-controlled microinfusion pump, as previously described. ${ }^{3}$ The catheter tip was placed stereotactically into the brainstem using coordinates derived from the Paxinos and Watson stereotactic rat brain atlas $(1.4 \mathrm{~mm}$ lateral to lambda, $-9 \mathrm{~mm}$ from bregma, and $8 \mathrm{~mm}$ deep from the dura).

Water-soluble panobinostat (MTX110, Midatech Pharma plc) at increasing concentrations, artificial CSF $(\mathrm{aCSF})$, or vehicle was administered at an infusion rate of $1 \mu \mathrm{l} / \mathrm{min}$ for 5 minutes (a $5-\mu \mathrm{l}$ volume of infusion) (Table 1). On completion of CED, the catheter was left in situ for 10 minutes to minimize reflux prior to slow catheter withdrawal $(1 \mathrm{~mm} / \mathrm{min})$. The wound was closed with $4 / 0$ Vicryl Rapide and analgesia (buprenorphine intramuscularly, $30 \mu \mathrm{g} / \mathrm{kg}$ ) administered prior to recovery.

\section{Striatal Infusion: Pharmacokinetic Analysis}

We infused $10 \mu \mathrm{l}$ of $300 \mu \mathrm{M}$ panobinostat in Wistar rats at $2.5 \mu \mathrm{l} / \mathrm{min}$ into the striatum using the following stereotactic landmarks: $0.75 \mathrm{~mm}$ rostral and $3 \mathrm{~mm}$ lateral to bregma, at a depth of $5.0 \mathrm{~mm}$ from the dura, through a 2-mm burr hole. The striatum was used for pharmacokinetic analysis as its larger anatomical target allowed easier dissection for tissue-drug concentration determination than the brainstem. Animals were euthanized by anesthetic overdose $0,0.5,2,6$, and 24 hours $(n=2$ per time point) after infusion, and their brains explanted and snap-frozen in liquid nitrogen (Table 2). Blood was taken from the right ventricle via cardiac puncture and stored in EDTA-containing tubes prior to freezing. Brains were dissected in half through the midline prior to freezing, 
TABLE 1. Small-animal experimental schedule for panobinostat CED: toxicity analysis

\begin{tabular}{ccccc}
\hline \multirow{2}{*}{$\begin{array}{c}\text { Group } \\
\text { No. }\end{array}$} & Group & $\begin{array}{c}\text { Concentration } \\
(\mu \mathrm{M})\end{array}$ & \multicolumn{2}{l}{ No. of Rats (time postinfusion) } \\
\cline { 4 - 5 } 1 & aCSF control & 0 & 3 & 14 Days \\
\hline 2 & Vehicle control & 0 & 3 & 3 \\
\hline 3 & Panobinostat & 1 & 3 & 3 \\
\hline 4 & Panobinostat & 3 & 3 & 3 \\
\hline 5 & Panobinostat & 10 & 3 & 3 \\
\hline 6 & Panobinostat & 30 & 3 & 3 \\
\hline
\end{tabular}

and drug concentration was determined by liquid chromatography tandem mass spectrometry (LC-MS/MS). A higher concentration was infused than that used for toxicity analysis to ensure that drug concentration within the brain was above quantifiable levels of detection at the 0 hours' time point.

\section{CED Procedure in Pigs}

Male juvenile Large White-Landrace cross pigs $(n=$ 4), approximately 12 weeks old (JA Moon) and weighing 40 to $45 \mathrm{~kg}$, were group-housed. The pigs were grouphoused in pens with straw from Andrew Duckett (Agricultural Product Supplies). Pen temperature was kept at a range of $17^{\circ} \mathrm{C}-22^{\circ} \mathrm{C}$.

A bespoke in-house-designed MRI-compatible head frame (Renishaw plc) was fixed to juvenile Large WhiteLandrace pigs under general anesthesia, as previously described.$^{1,4,15,30}$ A fiducial arc was then placed onto the frame, and the animal, while still under general anesthesia, was transferred to the MRI scanner (Philips field strength 1.5 T). Preoperative noncontrast T1 and T2 volume scans were obtained. A permanently implanted recessed-step catheter targeting the ventral upper pons in the midline from a frontal entry point was planned, using bespoke, in-house surgical planning software (NeuroInspire, Renishaw plc). A modified Cosman-Roberts-Wells stereotactic frame and arc was connected to the MRI-compatible frame, and catheters were implanted under sterile conditions using the stereotactic coordinates calculated from the preoperative plan. Once the catheters were implanted, the wound was closed and a second MR image was obtained before the animal recovered from anesthesia. The second MR image was fused to the preoperative image to verify accurate catheter placement. The surgical procedure and

TABLE 2. Small-animal experimental schedule to determine drug clearance after CED

\begin{tabular}{ccccc}
\hline $\begin{array}{c}\text { Group } \\
\text { No. }\end{array}$ & $\begin{array}{c}\text { Time } \\
\text { Postinfusion (hrs) }\end{array}$ & $\begin{array}{c}\text { Infused } \\
\text { Concentration }(\mu \mathrm{M})\end{array}$ & $\begin{array}{c}\text { Infused } \\
\text { Vol }(\mu \mathrm{l})\end{array}$ & $\begin{array}{c}\text { No. of } \\
\text { Animals }\end{array}$ \\
\hline 1 & 0 & 300 & 10 & 2 \\
\hline 2 & 2 & 300 & 10 & 2 \\
\hline 3 & 6 & 300 & 10 & 2 \\
\hline 4 & 24 & 300 & 10 & 2 \\
\hline
\end{tabular}

TABLE 3. Large-animal experimental schedule for panobinostat CED: toxicity analysis

\begin{tabular}{cclcc}
\hline $\begin{array}{c}\text { Animal } \\
\text { No. }\end{array}$ & $\begin{array}{c}\text { Weight } \\
(\mathrm{kg})\end{array}$ & \multicolumn{1}{c}{ Target } & $\begin{array}{c}\text { Infused Vol } \\
(\mu \mathrm{l})\end{array}$ & $\begin{array}{c}\text { Infused } \\
\text { Concentration }(\mu \mathrm{M})\end{array}$ \\
\hline 1 & 40 & Ventral pons & 100 & 30 \\
\hline 2 & 45 & Ventral pons & 100 & 30 \\
\hline 3 & 40 & Untreated control & NA & NA \\
\hline
\end{tabular}

$\mathrm{NA}=$ not applicable.

catheters are identical to those used in human treatment, making results directly translatable to the clinic. ${ }^{2}$

\section{Brainstem Infusion: Toxicity Analysis}

Physiological monitoring during surgery and drug infusion consisted of blood pressure, pulse, temperature and oxygen saturation monitoring, electrocardiography, and capnography. Two pigs received infusions of drug to the pons via an implanted recessed-step CED catheter (Table $3)$. Infusions were performed under general anesthesia. A fixed volume of drug was infused (100 $\mu \mathrm{l})$ using the following clinical ramping regime of infusion rate: $0.5 \mu \mathrm{l} /$ min for 10 minutes, $1.0 \mu \mathrm{l} / \mathrm{min}$ for 5 minutes, $2 \mu \mathrm{l} / \mathrm{min}$ for 5 minutes, and $3 \mu \mathrm{l} / \mathrm{min}$ until $100 \mu \mathrm{l}$ had been administered. An infusion of $100 \mu \mathrm{l}$ took 46.6 minutes.

Animals were examined daily for signs of neurological disability. Animals were euthanized by anesthetic overdose at either 7 or 14 days after drug administration $(n=1$ per time point); they then underwent transcardial paraformaldehyde perfusion fixation and brain explantation. The brains were stored in $4 \%$ paraformaldehyde prior to neuropathological examination. Treated animals were compared to an age- and weight-matched untreated control.

\section{Thalamic and Frontal White Matter Infusion: Drug Distribution Analysis}

A single pig had 2 drug delivery recessed-step catheters implanted. One catheter was implanted to target the frontal white matter and another into the contralateral thalamus (Table 4). These supratentorial structures were used for drug distribution analysis so that large volumes of drug could be administered without causing infusion volume-related neurological toxicity, which is more likely in the small porcine brainstem. A week after catheter implantation, the drug was infused under general anesthesia using the same ramping regime. At completion of the infusion, an MRI scan (volumetric T2 weighted and FLAIR) was obtained to determine the volume of infusion defined

TABLE 4. Infusion schedule for drug distribution analysis in pig brain

\begin{tabular}{ccccc}
\hline $\begin{array}{c}\text { Animal } \\
\text { No. }\end{array}$ & $\begin{array}{c}\text { Weight } \\
(\mathrm{kg})\end{array}$ & \multicolumn{1}{c}{ Target } & $\begin{array}{c}\text { Infused } \\
\text { Vol }(\mu \mathrm{l})\end{array}$ & $\begin{array}{c}\text { Infused } \\
\text { Concentration }(\mu \mathrm{M})\end{array}$ \\
\hline 4 & 46 & Frontal white matter & 1500 & 30 \\
\hline & & Thalamus $(\mathrm{GM})$ & 720 & 30 \\
\hline
\end{tabular}

$\mathrm{GM}=$ gray matter 


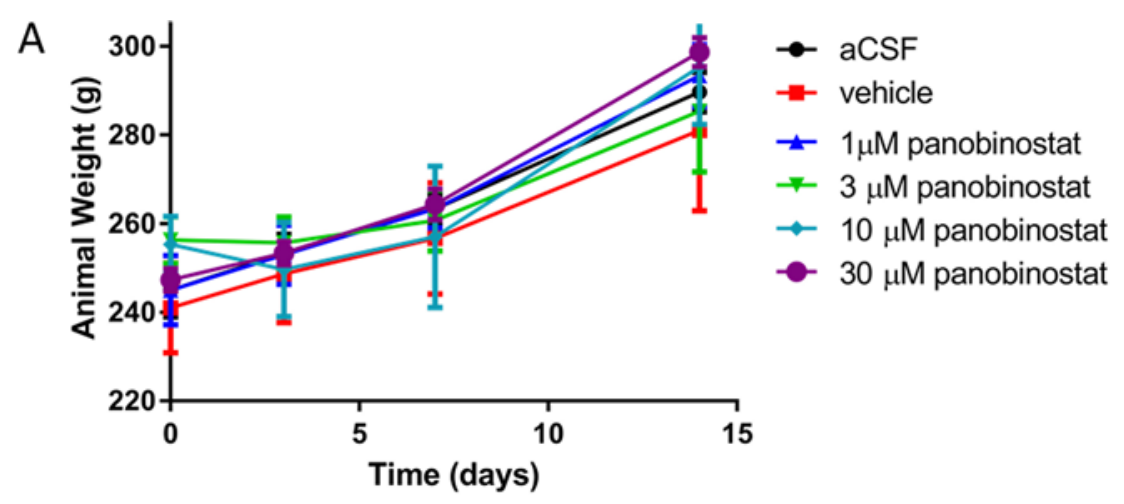

B

H\&E

GFAP aCSF control

NeuN

IBA-1
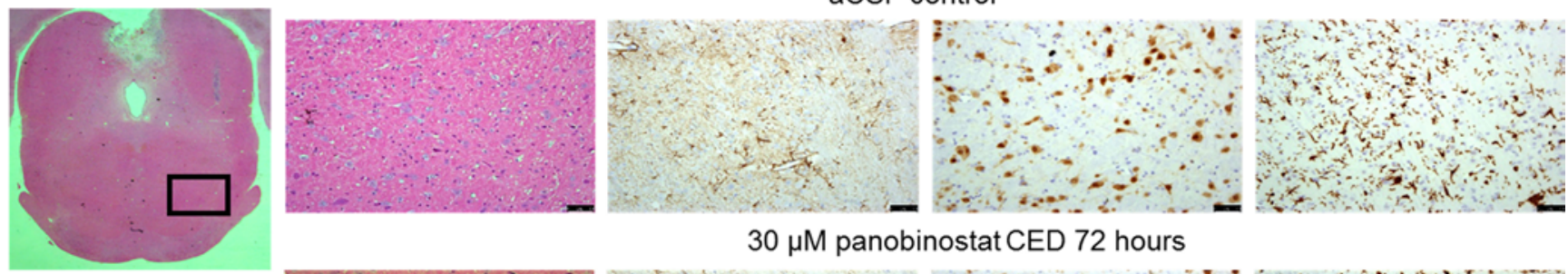

$30 \mu \mathrm{M}$ panobinostat CED 72 hours
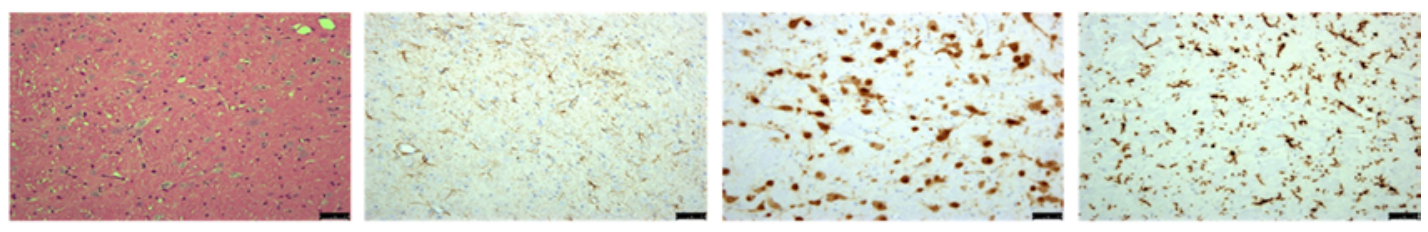

$30 \mu \mathrm{M}$ panobinostat CED 14 days
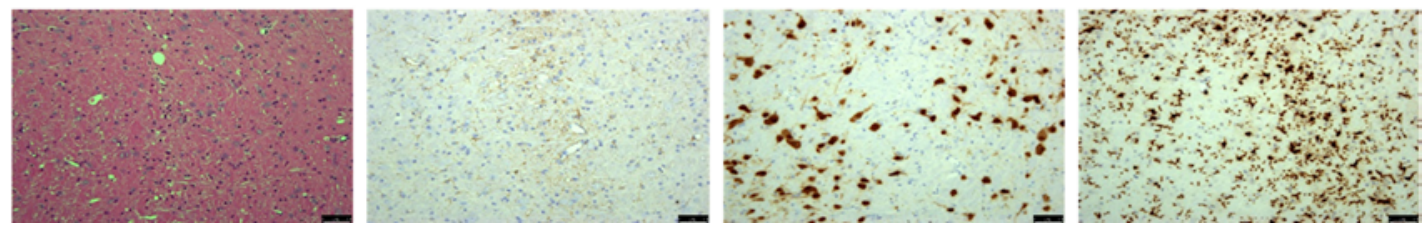

FIG. 1. Small-animal toxicity analysis. Following a dose-escalation study of water-soluble panobinostat, no difference was observed among groups of animals. A: Rat body weights after dose escalation of panobinostat CED to the brainstem ( 6 animals per group to 72 hours, and 3 animals per group to 14 days). No difference in animal weight gain was observed among groups of animals during the experimental period. B: Representative histological images from cohort of 36 juvenile Wistar rats, after blinded neuropathological examination. No morphological differences were seen across treated groups compared to vehicle-only controls. Bar $=20 \mu \mathrm{m}$.

by a change in $\mathrm{T} 2$ signal compared to that seen on preinfusion images. The animal was killed immediately following this, and the brain was explanted. The brain was then divided into right and left hemispheres. Each hemisphere was cut in serial coronal 1-cm blocks. Within each block, 6 small tissue sections were cut and snap-frozen in liquid nitrogen. Drug concentration in each section was determined by LC-MS/MS, and the position of each section was superimposed on the postinfusion MRI scan using stereotactic coordinates.

\section{Neuropathological Processing and Analysis}

All brains were cut into 4- to 5- $\mu \mathrm{m}$ coronal sections following wax embedding (Leica Microsystems). All sections for both immunohistochemistry and histopathological assessment were mounted on positively charged slides. $\mathrm{H} \& \mathrm{E}$ staining was performed using standard neuropathological techniques. Immunohistochemistry was used to assess damage to specific cell types. To identify neuronal disruption, gliosis, and activated microglia, neurons, astrocytes, and microglia were stained with the following primary antibodies: rabbit anti-NeuN (1:4000; Abcam), mouse anti-GFAP (1:800; Bio-Rad), and rabbit anti-Iba-1 (1:4000 Abcam). Iba-1 and NeuN antibody sections were subject to antigen retrieval using the Dako high-pH tissueretrieval solution in the Dako PT link. GFAP antibody sections were subject to antigen retrieval using Dako low$\mathrm{pH}$ tissue-retrieval solution. Secondary staining was performed using 5\% normal horse serum, anti-mouse (ratadsorbed) biotinylated secondary antibody, and streptavidin horseradish peroxidase (1:100) (Vector Labs). For all antibodies, the chromogen used was Liquid DAB (Dako) counterstained with hematoxylin (Dako).

Analysis of the tissue sections was performed by a neuropathologist who was blinded to the identification of experimental conditions. 

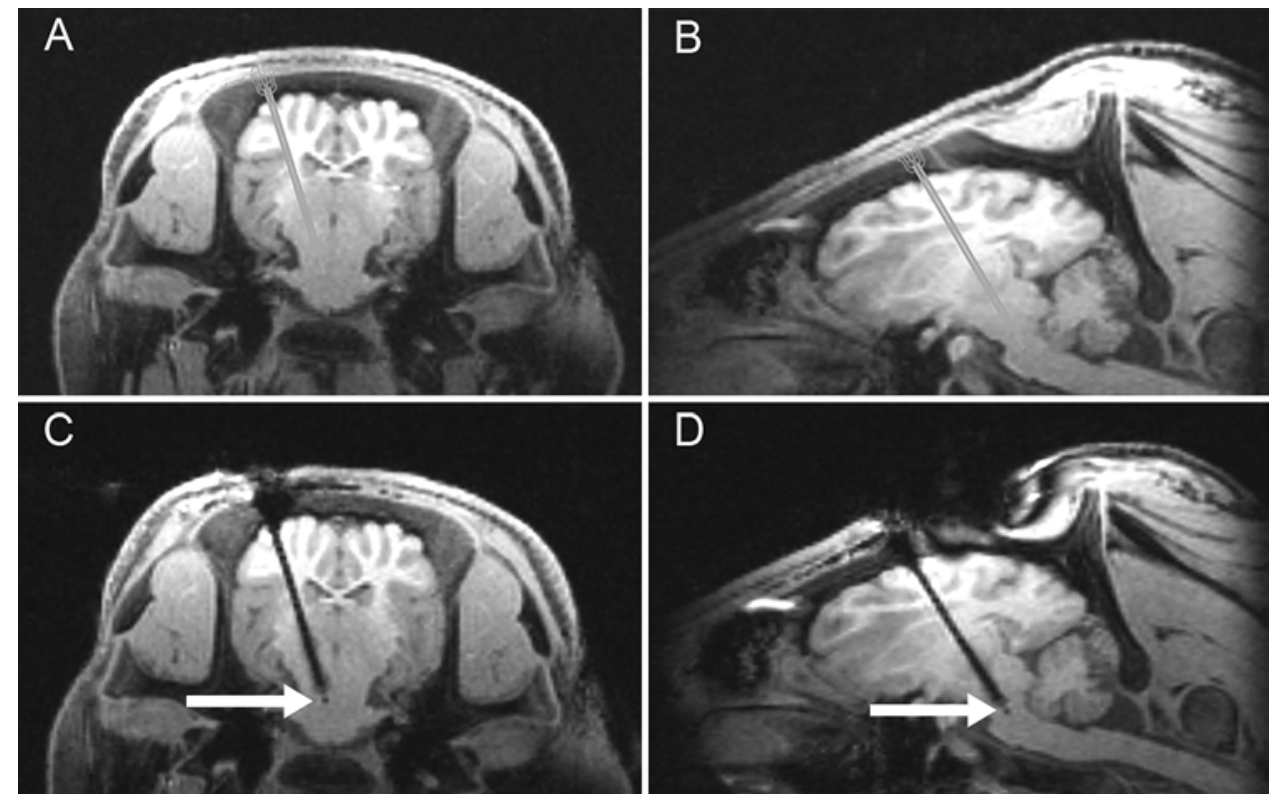

FIG. 2. Stereotactic implantation of skull-mounted, reflux-resistant, recessed-step Carbothane CED catheters into the ventral pons of juvenile Large White-Landrace cross pigs. Preoperative planning T1-weighted volumetric MR images: coronal plane (A), sagittal plane showing the planned catheter implant to the ventral upper pons using a transfrontal trajectory (B). Postoperative MR images showing the implanted recessed-step CED catheter at the planned target (white arrows) (C and D).

\section{LC-MS/MS Assay}

An LC-MS/MS assay for panobinostat in rat plasma and brain was developed to complete analysis of rat plasma and rat and pig brain samples. All samples were quantified against a panobinostat calibration line from 0.5 to $10,000 \mathrm{ng} / \mathrm{ml}$ using a Thermo TSQ Vantage mass spectrometer and Waters Aquity HPLC system. The HPLC conditions were as follows: column: $\mathrm{C} 18 \mathrm{BEH}, 1.7 \mu \mathrm{m}$, $50 \times 2.1 \mathrm{~mm}$; temperature: $50^{\circ} \mathrm{C}$; mobile phase organic: acetonitrile; mobile phase aqueous: $0.1 \%$ formic acid; and injection volume: $10 \mu \mathrm{l}$. The tandem mass spectroscopy details were as follows: spray voltage: 3000 ; vaporizer temperature: $350^{\circ} \mathrm{C}$; sheath gas pressure: $50 \mathrm{kPa}$; ion sweep gas pressure: $2 \mathrm{kPa}$; auxiliary gas pressure: $55 \mathrm{kPa}$; and capillary gas pressure: $214^{\circ} \mathrm{C}$.

Stock solutions of panobinostat were prepared in DMSO and accurately diluted to prepare the calibration standards. Quality control samples were prepared in triplicate using a stock reference solution. The method was tested using prepared control ex vivo rat brain and plasma infused with known concentration of panobinostat in DMSO. Tissue was homogenized prior to analysis.

\section{Results}

\section{Small-Animal Neurotoxicity}

A dose-escalation experiment was designed to infuse an increasing concentration of water-soluble panobinostat into the rat brainstem by CED (Table 1). Animals were treated in groups of 3 , and clinical observation continued for either 72 hours or 14 days after treatment. Brains were then subjected to neuropathological analysis. Alongside this, animals were treated in groups of 2 with infusion of water-soluble panobinostat to the left striatum and sacri- ficed at predetermined time points over a 24-hour period; the brains were explanted and snap-frozen to enable the drug concentration to be quantified on brain lysates by LC-MS/MS.

No clinical signs of toxicity were seen in any group compared to control animals. Their behavior, feeding pattern, and weight gain did not differ from those of animals treated with either vehicle or aCSF alone. Blinded neuropathological analysis did not reveal any morphological evidence of toxicity within the pons compared to control. NeuN-, GFAP-, and Iba-1-positive staining was present throughout the brain, but specifically no difference was observed within the target infusion area across the entire dose range (Fig. 1). The pattern of staining in brains treated with drug was not different from the pattern in those treated with vehicle or aCSF alone.

\section{Large-Animal Neurotoxicity}

Postoperative MRI indicated that the catheter position was within $1 \mathrm{~mm}$ of the target when fused to the preoperative MRI plan and was not associated with complications such as hemorrhage, which may have prevented drug infusion (Fig. 2). Drug infusions were completed without complication. Male juvenile Large White-Landrace cross pigs that received pontine CED of panobinostat $(n=2)$ had significant stiffness in their hind legs postoperatively that rapidly resolved over a 24-hour period and was attributed to their prone surgical positioning and prolonged anesthesia. There were no clinical or physiological signs of brainstem dysfunction in either animal during or after infusion. Both animals were eating and drinking well immediately after recovery from anesthesia. All other aspects of behavior and clinical examination were normal for the test period. 


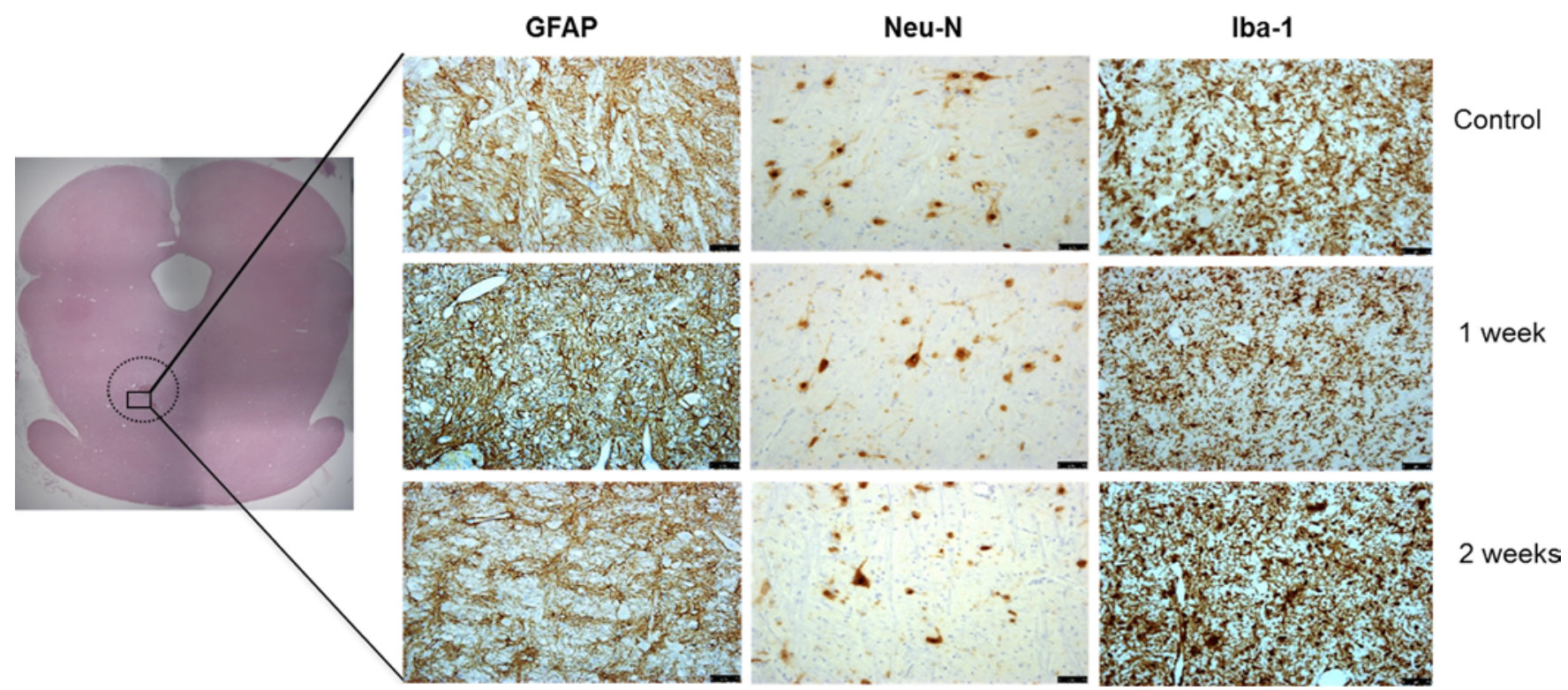

FIG. 3. Large-animal toxicity analysis. Neuropathology of pig brainstem after CED of $100 \mu$ l water-soluble panobinostat at a $30-$ $\mu \mathrm{M}$ concentration. No difference in tissue staining or morphology was seen at 1 and 2 weeks after CED compared to findings in untreated controls, all done by blinded neuropathological examination. Bar $=20 \mu \mathrm{m}$.

Neuropathological analysis of brainstem sections from explanted brains of animals receiving water-soluble panobinostat CED to the ventral pons was unremarkable compared to control tissue. There was evidence of local trauma at the site of catheter placement. There was no difference in the staining pattern of glia, microglia, or neurons within the target infusion area at the catheter tip within the pons of either the 1- or 2-week animals (Fig. 3). Blinded pathological analysis did not reveal any evidence of toxicity within the brains of treated animals.

\section{Panobinostat Clearance From Rat Striatum After CED}

Water-soluble panobinostat cleared rapidly from rat

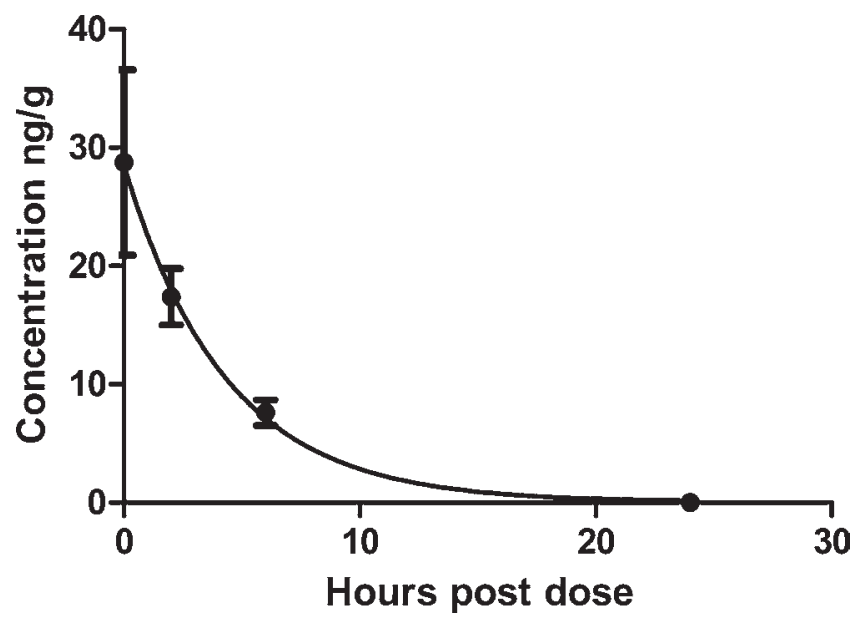

FIG. 4. Half-life of panobinostat in brain parenchyma after CED. The tissue concentration of water-soluble panobinostat was determined by LC-MS/MS after acute infusion by CED into the rat striatum (triplicate results, error bars indicate SEM). Calculated tissue half-life was 2.9 hours. striatum. Panobinostat was detectable in brain tissue by LC-MS/MS at quantifiable levels 0,2 , and 6 hours postinfusion but not after. Half-life in brain parenchyma after CED was calculated to be 2.9 hours (Fig. 4). No drug was detectable in plasma at any time point after CED.

\section{Distribution of Panobinostat in Pig Brain After Acute CED}

The volume of T2 signal change at the end of thalamus and frontal white matter infusion was calculated from real-time 3D volumetric T2-weighted MRI scans. The volume of T2 signal change in the thalamus and frontal white matter was $2697 \mathrm{~mm}^{3}$ and $3108 \mathrm{~mm}^{3}$, respectively. After acute infusion of water-soluble panobinostat to the thalamus and frontal white matter, panobinostat was detectable in brain tissue within the entire region of signal change on the real-time intra-infusion T2-weighted MRI as detected by LC-MS/MS. This indicated that water-soluble panobinostat distributed after direct intraparenchymal infusion in a pattern that mirrored the change in T2 signal (Fig. 5). The volume of infusion to volume of distribution ratio (Vi/ Vd) was 1:2 and 1:3 in white and gray matter, respectively, ratios that are consistent with drug distribution by convection.

\section{Discussion}

We have shown that panobinostat (MTX110) in increasing concentrations up to $30 \mu \mathrm{M}$ does not cause neurotoxicity 72 hours and 14 days after administration by CED in a single dose to the rat pons. The same highest dose was not toxic to porcine brainstem 7 and 14 days after infusion of a clinically relevant volume of drug. Further preclinical analysis to determine the toxic infused concentration of panobinostat would require extension of this study to incorporate higher concentration of drug. The doses used in 

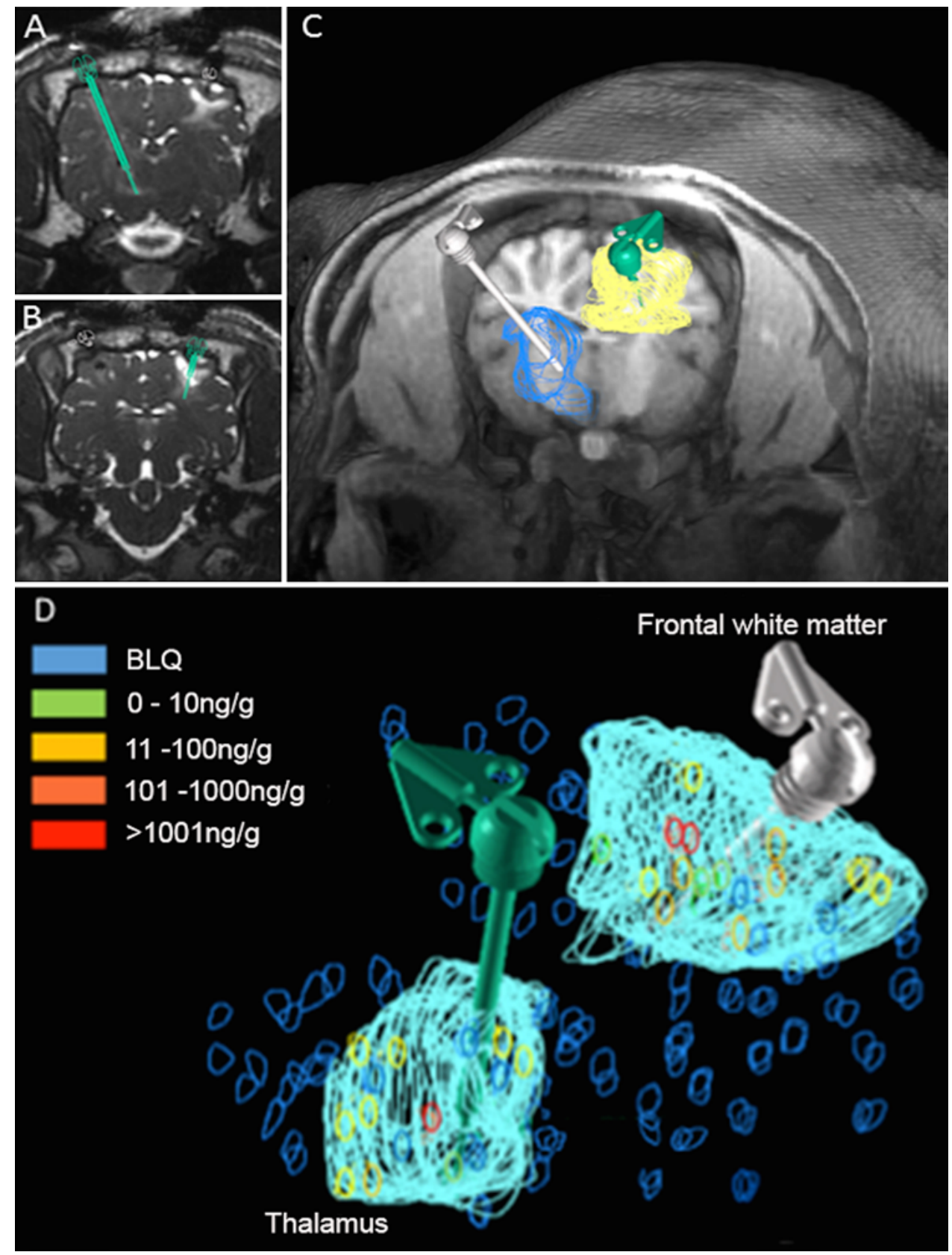

FIG. 5. Water-soluble panobinostat distribution in white and gray matter after CED. Real-time T2-weighted volumetric 3D MRI during panobinostat CED in the pig brain. Catheter plans (green) are superimposed onto the intra-infusion T2-weighted MR images of a thalamic catheter $(\mathbf{A})$ and frontal white matter catheter $(\mathbf{B})$. Volume analysis of the change in T2 signal on an intra-infusion MR image in 3D (C); the volume of T2 signal change is outlined in yellow (frontal white matter) and blue (thalamus). Panobinostat was detected by LC-MS/MS throughout the volume of T2 signal change on a real-time intra-infusion MR image (D). Tissue samples taken from explanted brain were analyzed for the presence and concentration of panobinostat. The stereotactic coordinates of the position of these biopsies were superimposed on the 3D intra-infusion volume MR image and colored according to the drug concentration. Drug was detectable throughout the area of brain infused as defined by the change in T2 signal (3D volume outlined in light blue). The highest drug concentration was detectable adjacent to the catheter tips. Drug concentration color coded as shown. Green catheter $=$ thalamus; white catheter $=$ frontal white matter. $\mathrm{BLQ}=$ below level of quantification.

this study are far in excess of those shown to be effective in preclinical models of DIPG, both in vitro and in vivo.

Drug distribution analysis illustrated that panobinostat distributed within white and gray matter in a large gyrencephalic brain at a volume of distribution greater than the volume of infusion, indicating that the drug distributes by convection, which supports the finding in this formulation that panobinostat is likely to distribute effectively in human brain when administered by CED. The half-life of panobinostat administered by single-infusion CED is 
short in the rat brain. The rate of cerebral blood flow is much higher in the rat brain than in the human brain, and therefore one would expect the half-life of infused panobinostat to be longer in the human brain after CED. Despite this, the tissue-drug half-life is likely to still be rapid in humans. Repeated administration to a tumor may be required to ensure an accurate investigation of drug efficacy within a CED clinical trial. This could be achieved by using an implantable drug delivery system that allows repeat administration of the drug by CED without the requirement of repeat surgery. ${ }^{2,27}$

\section{Conclusions}

The findings in this study support the use of watersoluble panobinostat administered by CED. There was no toxicity observed in the experiments performed at concentrations far more than those effective against DIPG in vitro. ${ }^{11,13}$ No clinically relevant DIPG animal model that is suitable to accurately evaluate the efficacy or clinical toxicity of drugs administered by CED exists, so this additional analysis prior to clinical translation is not currently possible. The results of the experiments presented here provide reassuring data that the use of water-soluble panobinostat (MTX110) administered by CED to a dose of $30 \mu \mathrm{M}$ (10.47 $\mu \mathrm{g} / \mathrm{ml})$ is not associated with neurotoxicity and therefore supports the translation of the therapy to the clinic, with a rationale for a starting dose in an early-phase trial.

Panobinostat shows preclinical efficacy against both DIPG and non-brainstem high-grade glioma, including adult glioblastoma. The oral formulation of the drug has failed to show efficacy in clinical trials potentially because of poor CNS penetration across the BBB from the systemic circulation. ${ }^{11,17,22}$ Panobinostat is insoluble in water in its current clinical formulation, which means that oral dosing is currently the only possible method of administration. In the context of a carefully designed concentrationescalation clinical trial, CED of new water-soluble forms of panobinostat could be a novel treatment strategy for patients with DIPG and also for those with supratentorial high-grade glioma.

\section{Acknowledgments}

Medical Research Council/The Brain Tumour Charity, UK Clinical Research Training Fellowship (grant MR/N00130/1).

\section{References}

1. Arshad A, Yang B, Bienemann AS, Barua NU, Wyatt MJ, Woolley M, et al: Convection-enhanced delivery of carboplatin PLGA nanoparticles for the treatment of glioblastoma. PLoS One 10:e0132266, 2015

2. Barua NU, Hopkins K, Woolley M, O'Sullivan S, Harrison R, Edwards RJ, et al: A novel implantable catheter system with transcutaneous port for intermittent convection-enhanced delivery of carboplatin for recurrent glioblastoma. Drug Deliv 23:167-173, 2016

3. Barua NU, Woolley M, Bienemann AS, Johnson D, Wyatt MJ, Irving C, et al: Convection-enhanced delivery of AAV2 in white matter-a novel method for gene delivery to cerebral cortex. J Neurosci Methods 220:1-8, 2013

4. Barua NU, Woolley M, Bienemann AS, Johnson DE, Lewis $\mathrm{O}$, Wyatt MJ, et al: Intermittent convection-enhanced de- livery to the brain through a novel transcutaneous boneanchored port. J Neurosci Methods 214:223-232, 2013

5. Bienemann A, White E, Woolley M, Castrique E, Johnson DE, Wyatt M, et al: The development of an implantable catheter system for chronic or intermittent convection-enhanced delivery. J Neurosci Methods 203:284-291, 2012

6. Bobo RH, Laske DW, Akbasak A, Morrison PF, Dedrick RL, Oldfield EH: Convection-enhanced delivery of macromolecules in the brain. Proc Natl Acad Sci U S A 91:2076-2080, 1994

7. Buczkowicz P, Hawkins C: Pathology, molecular genetics, and epigenetics of diffuse intrinsic pontine glioma. Front Oncol 5:147, 2015

8. Castel D, Philippe C, Calmon R, Le Dret L, Truffaux N, Boddaert N, et al: Histone H3F3A and HIST1H3B K27M mutations define two subgroups of diffuse intrinsic pontine gliomas with different prognosis and phenotypes. Acta Neuropathol 130:815-827, 2015

9. Gill T, Barua NU, Woolley M, Bienemann AS, Johnson DE, O'Sullivan S, et al: In vitro and in vivo testing of a novel recessed-step catheter for reflux-free convection-enhanced drug delivery to the brain. J Neurosci Methods 219:1-9, 2013

10. Goodwin CR, Xu R, Iyer R, Sankey EW, Liu A, Abu-Bonsrah $\mathrm{N}$, et al: Local delivery methods of therapeutic agents in the treatment of diffuse intrinsic brainstem gliomas. Clin Neurol Neurosurg 142:120-127, 2016

11. Grasso CS, Tang Y, Truffaux N, Berlow NE, Liu L, Debily MA, et al: Functionally defined therapeutic targets in diffuse intrinsic pontine glioma. Nat Med 21:555-559, 2015 (Erratum in Nat Med 21:827, 2015)

12. Halvorson KG, Barton KL, Schroeder K, Misuraca KL, Hoeman C, Chung A, et al: A high-throughput in vitro drug screen in a genetically engineered mouse model of diffuse intrinsic pontine glioma identifies BMS-754807 as a promising therapeutic agent. PLoS One 10:e0118926, 2015

13. Hennika T, Hu G, Olaciregui NG, Barton KL, Ehteda A, Chitranjan A, et al: Pre-clinical study of panobinostat in xenograft and genetically engineered murine diffuse intrinsic pontine glioma models. PLoS One 12:e0169485, 2017

14. Jansen MH, Veldhuijzen van Zanten SE, Sanchez Aliaga E, Heymans MW, Warmuth-Metz M, Hargrave D, et al: Survival prediction model of children with diffuse intrinsic pontine glioma based on clinical and radiological criteria. Neuro Oncol 17:160-166, 2015

15. Jones A, Bienemann A, Barua N, Murison PJ, Gill S: Anaesthetic complications in pigs undergoing MRI guided convection enhanced drug delivery to the brain: a case series. Vet Anaesth Analg 39:647-652, 2012

16. Khuong-Quang DA, Buczkowicz P, Rakopoulos P, Liu XY, Fontebasso AM, Bouffet E, et al: K27M mutation in histone H3.3 defines clinically and biologically distinct subgroups of pediatric diffuse intrinsic pontine gliomas. Acta Neuropathol 124:439-447, 2012

17. Lee EQ, Reardon DA, Schiff D, Drappatz J, Muzikansky A, Grimm SA, et al: Phase II study of panobinostat in combination with bevacizumab for recurrent glioblastoma and anaplastic glioma. Neuro Oncol 17:862-867, 2015

18. Luther N, Zhou Z, Zanzonico P, Cheung NK, Humm J, Edgar MA, et al: The potential of theragnostic ${ }^{124} \mathrm{I}-8 \mathrm{H} 9$ convectionenhanced delivery in diffuse intrinsic pontine glioma. Neuro Oncol 16:800-806, 2014

19. Morales La Madrid A, Hashizume R, Kieran MW: Future clinical trials in DIPG: bringing epigenetics to the clinic. Front Oncol 5:148, 2015

20. Morrison PF, Laske DW, Bobo H, Oldfield EH, Dedrick RL: High-flow microinfusion: tissue penetration and pharmacodynamics. Am J Physiol 266:R292-R305, 1994

21. Nikbakht H, Panditharatna E, Mikael LG, Li R, Gayden T, Osmond M, et al: Spatial and temporal homogeneity of driver 
mutations in diffuse intrinsic pontine glioma. Nat Commun 7:11185, 2016

22. Pont LM, Naipal K, Kloezeman JJ, Venkatesan S, van den Bent M, van Gent DC, et al: DNA damage response and anti-apoptotic proteins predict radiosensitization efficacy of HDAC inhibitors SAHA and LBH589 in patient-derived glioblastoma cells. Cancer Lett 356 (2 Pt B):525-535, 2015

23. Rasmussen TA, Tolstrup M, Møller HJ, Brinkmann CR, Olesen R, Erikstrup C, et al: Activation of latent human immunodeficiency virus by the histone deacetylase inhibitor panobinostat: a pilot study to assess effects on the central nervous system. Open Forum Infect Dis 2:ofv037, 2015

24. Rodgers L, McCully CL, Peer C, Cruz R, Figg W: Plasma and cerebrospinal fluid (CSF) pharmacokinetics of panobinostat following oral administration to nonhuman primates. Neuro Oncol 18:iii55, 2016 (Abstract)

25. Saito R, Krauze MT, Noble CO, Tamas M, Drummond DC, Kirpotin DB, et al: Tissue affinity of the infusate affects the distribution volume during convection-enhanced delivery into rodent brains: implications for local drug delivery. J Neurosci Methods 154:225-232, 2006

26. Singleton WG, Collins AM, Bienemann AS, Killick-Cole CL, Haynes HR, Asby DJ, et al: Convection enhanced delivery of panobinostat (LBH589)-loaded pluronic nano-micelles prolongs survival in the F98 rat glioma model. Int J Nanomedicine 12:1385-1399, 2017

27. Singleton WGB, Barua NU, Morgan J, Bienemann AS, Killick-Cole CL, Asby DJ, et al: Multi-catheter intermittent convection-enhanced delivery of carboplatin as a treatment for diffuse intrinsic pontine glioma (DIPG): pre-clinical rationale and early clinical experience. Neuro Oncol 18:iii131, 2016 (Abstract)

28. Veldhuijzen van Zanten SE, Jansen MH, Sanchez Aliaga E, van Vuurden DG, Vandertop WP, Kaspers GJ: A twenty-year review of diagnosing and treating children with diffuse intrinsic pontine glioma in The Netherlands. Expert Rev Anticancer Ther 15:157-164, 2015

29. Veringa SJ, Biesmans D, van Vuurden DG, Jansen MH, Wedekind LE, Horsman I, et al: In vitro drug response and efflux transporters associated with drug resistance in pediatric high grade glioma and diffuse intrinsic pontine glioma. PLoS One 8:e61512, 2013

30. White E, Woolley M, Bienemann A, Johnson DE, Wyatt M, Murray G, et al: A robust MRI-compatible system to facilitate highly accurate stereotactic administration of therapeutic agents to targets within the brain of a large animal model. J Neurosci Methods 195:78-87, 2011

31. Williams MJ, Singleton WGB, Lowis SP, Malik K, Kurian KM: Therapeutic targeting of histone modifications in adult and pediatric high-grade glioma. Front Oncol 7:45, 2017

32. Zhou Z, Singh R, Souweidane MM: Convection-enhanced delivery for diffuse intrinsic pontine glioma treatment. Curr Neuropharmacol 15:116-128, 2017

\section{Disclosures}

S.S.G. and A.S.B. are consultant advisors to Renishaw plc. M.W., D.J., and O.L. are employees of Renishaw plc. S.J.P.D. is an employee of Midatech Pharma plc. Study costs were sponsored by Midatech Pharma plc and Renishaw plc.

\section{Author Contributions}

Conception and design: Gill, Singleton, Bienemann. Acquisition of data: Singleton, Bienemann, Wyatt, Boulter. Analysis and interpretation of data: Gill, Singleton, Bienemann, Killick-Cole, Asby. Drafting the article: Singleton. Critically revising the article: Gill, Singleton, Bienemann, Woolley, Wyatt, Boulter, Killick-Cole, Asby. Reviewed submitted version of manuscript: Gill, Singleton, Bienemann, Woolley, Johnson, Lewis, Wyatt, Damment, Killick-Cole, Asby. Approved the final version of the manuscript on behalf of all authors: Gill. Administrative/technical/material support: Singleton, Woolley, Johnson, Lewis, Wyatt, Damment, Boulter. Study supervision: Gill.

\section{Supplemental Information}

\section{Previous Presentations}

Portions of this work were presented, and published in abstract form, at the 4th Pediatric Neuro-Oncology Basic and Translational Research Conference, June 15-16, 2017, New York, NY, and at the 22nd Annual Scientific Meeting and Education Day of the Society for Neuro-Oncology, November 16-19, 2017, San Francisco, CA.

\section{Correspondence}

Steven Gill: Clinical Neurosciences, Translational Health Sciences, University of Bristol, Southmead Hospital, Bristol, United Kingdom. steven.gill@nbt.nhs.uk. 